\title{
DETERMINAÇÃO POTENCIOMÉTRICA DA CONCENTRAÇÃO MICELAR CRÍTICA DE SURFACTANTES: UMA NOVA APLICAÇÃO METODOLÓGICA NO ENSINO DE QUÍMICA
}

\author{
Ivanise $M^{\mathrm{a}}$ Rizzatti* e Dilson R. Zanette \\ Departamento de Química, Centro de Ciências Físicas e Matemáticas, Universidade Federal de Santa Catarina, 88040-900 \\ Florianópolis - SC, Brasil \\ Ligia C. Mello \\ Colégio de Aplicação, Centro de Educação, Universidade Federal de Santa Catarina, 88040-900 Florianópolis - SC, Brasil \\ Recebido em 16/11/07; aceito em 18/8/08; publicado na web em 5/2/09
}

\begin{abstract}
POTENCIOMETRIC DETERMINATION OF THE CRITICAL MICELLAR CONCENTRATION OF THE SURFACTANTS: A NEW METHODOLOGICAL APPLICATION IN THE CHEMICAL LEARNING. The participation of the students in the planning, execution and discussion of experimental results is important for the valuation of the role of them in the construction of the chemical knowledge implicited in the approach between education and research. This work relates either the construction of selective membrane electrodes for surfactants and its application in the potenciometric determination of the critical micellar concentration of the sodium dodecyl sulfate (SDS) and dodecyltrimethylammonium bromide (DTAB) surfactants. In adition it's possible to discuss the potenciometric results in matching with the data valued for the tensiometric and condutimetric traditionally used in the physicalchemistry lessons.
\end{abstract}

Keywords: surfactants; potentiometry; chemistry education.

\section{INTRODUÇÃO}

Surfactantes ou agentes tensoativos são moléculas anfifílicas, que se associam espontaneamente em solução aquosa, a partir de uma determinada concentração. Apresentam uma região apolar e outra polar, ou iônica, e podem ser divididos em neutros ou iônicos. Os tensoativos iônicos podem ser catiônicos ou aniônicos ou, ainda, anfóteros, quando ambas as cargas estão presentes no surfactante. ${ }^{1}$

Em soluções diluídas as moléculas dos surfactantes apresentam-se dispersas, enquanto que em soluções mais concentradas organizamse em agregados moleculares, que geralmente contêm de 50 a 100 moléculas, denominados micelas.

A principal razão que leva os monômeros de surfactantes a se associarem sob a forma de micelas é a diminuição da área de contato entre as cadeias hidrocarbônicas do surfactante e da água. ${ }^{1}$

A concentração onde se inicia o processo de formação das micelas, micelização, é denominada concentração micelar crítica $(\mathrm{cmc})$, que é uma propriedade intrínseca e característica do surfactante a uma dada temperatura e concentração eletrolítica. ${ }^{2}$ São as micelas as responsáveis pela catálise micelar e pela solubilização de gorduras.

Durante a formação dos agregados, os grupos hidrofílicos (cabeças) dos surfactantes ficam muito próximos, gerando uma repulsão eletrostática que se opõe ao processo de micelização, conforme ilustrado na Figura 1. Nessa etapa, os contra-íons desempenham um papel fundamental: blindam a carga do agregado e diminuem o potencial elétrico, provocando repulsão entre as cabeças dos monômeros. ${ }^{1,2}$

$\mathrm{O}$ processo de formação dos agregados ocorre num intervalo pequeno de concentrações e pode ser detectado pela variação brusca produzida em determinadas propriedades físico-químicas da solução em função da concentração do surfactante, como mostrado na Figura 2. ${ }^{3}$

Os métodos normalmente utilizados na determinação de cmc de surfactantes são a condutimetria e a tensiometria. Neste trabalho propõe-se utilizar a potenciometria como alternativa ao estudo de surfactantes.

*e-mail: niserizzatti@hotmail.com

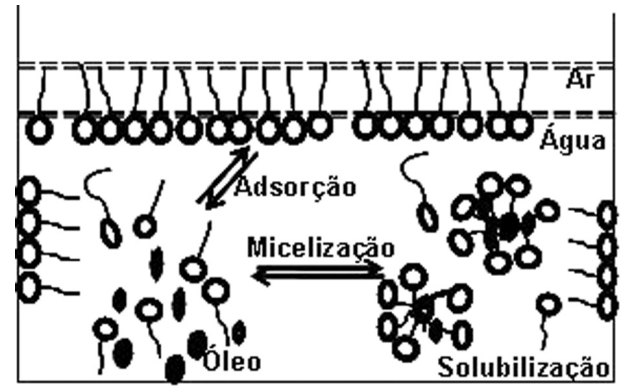

Figura 1. Ilustração dos fenômenos de adsorção líquido-gás, líquidosólido, formação de micelas e solubilização que ocorrem em soluções de surfactantes

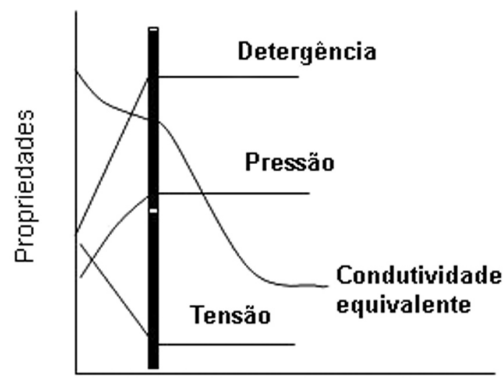

Concentração do surfactante

Figura 2. Representação de algumas propriedades físico-químicas em função da concentração de surfactante. A área hachureada corresponde à cmc

\section{Potenciometria}

Os métodos potenciométricos são baseados nas medidas do potencial das células eletroquímicas na ausência de correntes apreciáveis. Desde o início deste século, técnicas potenciométricas são utilizadas 
para a localização dos pontos finais nas análises por métodos titulométricos. ${ }^{4}$ A potenciometria também tem sido utilizada como um método pelo qual as concentrações dos íons são obtidas diretamente do potencial de um eletrodo de membrana seletivo a íon. Tais eletrodos são relativamente livres de interferência e proporcionam medições rápidas e convenientes para determinações quantitativas de cátions e ânions..$^{5} \mathrm{O}$ desenvolvimento da potenciometria em eletrodos seletivos a íons é uma área de grande interesse, sendo utilizada em larga escala para a determinação de íons em ambientes aquosos. ${ }^{6}$

Eletrodos seletivos a íons são definidos como sensores eletroquímicos que monitoram variações de atividade iônica em solução, sendo o potencial do eletrodo descrito pela Equação de Nernst ${ }^{4}$ (Equação 1):

$$
\mathrm{E}=\mathrm{E}^{\mathrm{o}} \pm(0,0592 / \mathrm{n}) \cdot \log a_{\mathrm{A}}
$$

onde, E representa o potencial do eletrodo íon seletivo; $\mathrm{E}^{\circ}$, o potencial padrão do eletrodo; $\mathrm{n}$ corresponde à carga iônica do íon e $a_{A}$, à atividade do íon primário, para o qual o eletrodo responde. Convenciona-se utilizar coeficiente angular positivo para cátions, e negativo para ânions. ${ }^{7}$

Os eletrodos indicadores de membrana, essenciais para a prática da análise potenciométrica, baseiam-se em reações de troca iônica e difusão de íons nas interfaces de diferentes tipos de membrana.,

O eletrodo de membrana utilizado neste trabalho, para o estudo de surfactantes, é constituído de um trocador líquido imobilizado em membrana de cloreto de polivinila (PVC), dissolvidos em tetraidrofurano (THF). ${ }^{1}$ A evaporação do solvente produz uma membrana flexível, que pode ser cortada e fixada na extremidade de um tubo plástico ou de vidro.

Neste artigo são apresentados os resultados da aplicação de uma metodologia para obtenção da concentração micelar crítica $(\mathrm{cmc})$ dos tensoativos dodecilsulfato de sódio (SDS) e brometo de dodeciltrimetilamônio (DTAB). Além disso, a exemplo de outros trabalhos citados na literatura, ${ }^{8,9}$ a utilização da potenciometria reforça novas tendências de pesquisa que permitem aos estudantes aprofundar conhecimentos a respeito do comportamento de eletrodos modificados, executando experimentos simples, com materiais acessíveis e de baixo custo.

\section{CONTRIBUIÇÕES PEDAGÓGICAS DA EXPERIMENTAÇÃO NO ENSINO DE QUÍMICA}

As atividades experimentais em geral têm sido enfatizadas, dada a sua importância do ponto de vista do desenvolvimento cognitivo e da utilização do método científico na resolução de problemas. ${ }^{10}$

No entanto, nos cursos de Química, em geral, prevalece uma visão simplista sobre a experimentação, muitas vezes embasada no empirismo da observação e teorização. É comum o entendimento de que a demonstração ou comprovação de um fenômeno é suficiente para a aprendizagem.

Torna-se necessário, então, que se proceda a um planejamento de atividades experimentais com o objetivo de discutir e aprofundar o conhecimento sobre a natureza da Ciência. Conhecimento este que influencia a aprendizagem dos estudantes ${ }^{11} \mathrm{e}$ auxilia na compreensão de que as observações estão relacionadas com as teorias e possibilitam uma interpretação das mesmas. ${ }^{12}$

Outro aspecto a ser ressaltado, quanto às atividades experimentais, é o papel motivador que desempenham. Apesar de alguns estudos confirmarem as expectativas de professores e alunos a respeito da motivação inerente às aulas experimentais, admite-se que provavelmente os alunos muitas vezes se motivam justamente pelo aspecto visual dos experimentos.

Contudo, uma das características positivas das atividades ex- perimentais é a possibilidade de questionamentos e explicitação do conhecimento dos alunos. Desse modo, desde que realizados de forma criativa, por meio de atividades e resolução de problemas relacionados ao cotidiano dos alunos, os experimentos deixam de ser uma forma de comprovação de conhecimentos e passam a atuar como uma atividade inovadora, encorajando o questionamento e a formulação de hipóteses que revelem o caráter contraditório do conhecimento. Ou seja, cria-se para o aluno uma situação de dificuldade intelectual conhecida como situação-problema, que deve ter como consequiência a passagem por diferentes níveis de entendimento dos fenômenos estudados. ${ }^{13}$

Torna-se evidente a importância da participação dos educandos no contexto das aulas de laboratório dos cursos de graduação em Química, durante o planejamento e estruturação do trabalho experimental, pois promove a reconstrução do conhecimento e confere à universidade o papel de centro de competência na aproximação entre pesquisa e educação. ${ }^{11,14}$

A metodologia proposta neste trabalho consiste no desenvolvimento e aplicação de eletrodos de membrana construídos em laboratório, destacando a potenciometria como uma alternativa às técnicas clássicas de condutimetria e tensiometria, usualmente empregadas nas aulas de físico-química dos cursos de graduação.

Paralelamente, ao discutir a importância de um experimento simples, em que a técnica potenciométrica pode ser comparada às técnicas usuais, pretende-se também enfatizar o papel da experimentação nas aulas de físico-química, como forma de estimular os estudantes a atuarem na pesquisa, construindo conhecimentos múltiplos da área de Química.

\section{PARTE EXPERIMENTAL}

\section{Construção dos eletrodos seletivos para determinação da cmc}

\section{Materiais e reagentes}

Utilizaram-se os reagentes dodecilsulfato de sódio (SDS), proveniente da Sigma, brometo de dodeciltrimetilamônio (DTAB) e plastificante dibutilftalato, da Aldrich, na forma em que foram recebidos, e os reagentes cloreto de polivinila (PVC), brometo de sódio, nitrato de prata e sulfeto de sódio, de procedência Sigma. As soluções foram preparadas com água desionizada pelo sistema água Millipore Milli-Q.

\section{Eletrodo de membrana seletiva para surfactante}

A mistura de surfactantes foi preparada a partir de 1,4220 g de SDS e 1,5415 g de DTAB, dissolvidos separadamente em béquer contendo $10 \mathrm{~mL}$ de água desionizada. As soluções foram misturadas resultando num precipitado branco, que foi filtrado e lavado várias vezes com água desionizada, recristalizado em acetona a quente, filtrado e seco em dessecador.

A membrana polimérica foi obtida a partir de $1,0 \mathrm{~g}$ de PVC, 0,0012 g do precipitado e 1,5 g de dibutilftalato de potássio, dissolvidos em $10 \mathrm{~mL}$ de THF, sob agitação constante. ${ }^{1}$ Após a evaporação do solvente, a membrana foi cortada e fixada no topo de um tubo de vidro de $1 \mathrm{~cm}$ de diâmetro por $10 \mathrm{~cm}$ de comprimento. Internamente um fio de prata recoberto com brometo de prata foi imerso em solução $0,01 \mathrm{~mol} \mathrm{~L}^{-1}$ de $\mathrm{NaBr}$ e SDS.

\section{Eletrodo de membrana sólida para brometo}

O eletrodo de referência consiste de um tubo de vidro de $10 \mathrm{~cm}$ de comprimento e $1 \mathrm{~cm}$ de diâmetro, onde foi fixada uma membrana sólida seletiva para brometo nas proporções 2:1 de $\mathrm{AgBr} / \mathrm{Ag}_{2} \mathrm{~S}$ prensada a 25-30 t. ${ }^{10}$ Internamente foi imerso um fio de prata recoberto com brometo de prata em solução $0,01 \mathrm{~mol} \mathrm{~L}^{-1}$ de $\mathrm{NaBr}^{1,9}$ 


\section{Técnicas utilizadas para a determinação da cmc}

Para determinar a cmc dos surfactantes SDS e DTAB utilizaramse três técnicas distintas: Condutimétrica - titulações condutimétricas foram realizadas a $25^{\circ} \mathrm{C}$, com um condutivímetro da marca Atiorion modelo 170 . O procedimento de medição consiste da adição de uma solução estoque de surfactante numa célula que contém um volume apropriado de água destilada e desmineralizada mantida sob agitação. A cmc foi obtida do encontro de duas retas do gráfico de condutividade em função da concentração de SDS. Todas as correlações lineares forneceram valores maiores que 0,999; Tensiométrica - as medidas de tensão superficial foram obtidas utilizando um tensiômetro da Kruss GMBH, modelo $\mathrm{K} 8$, com escala de divisão de $0,1 \mathrm{mNm}$ equipado com um anel de Pt-Ir-20. Para cada medição, foi utilizado volume de $10 \mathrm{~mL}$ da solução do surfactante correspondente em um frasco termostatizado a $25{ }^{\circ} \mathrm{C}$; Potenciométrica - o sistema dispõe de um potenciômetro da marca Orion 720A, um eletrodo de membrana seletiva para surfactante (indicador) e eletrodo para brometo. ${ }^{9}$ As medições de potencial foram obtidas a $25^{\circ} \mathrm{C}$, após adição de volume previamente calculado de surfactante. A cmc é determinada através da construção da curva de potencial em função do logaritmo da concentração molar de surfactante.

A Figura 3 ilustra o sistema utilizado para obtenção das medidas potenciométricas e os eletrodos empregados.

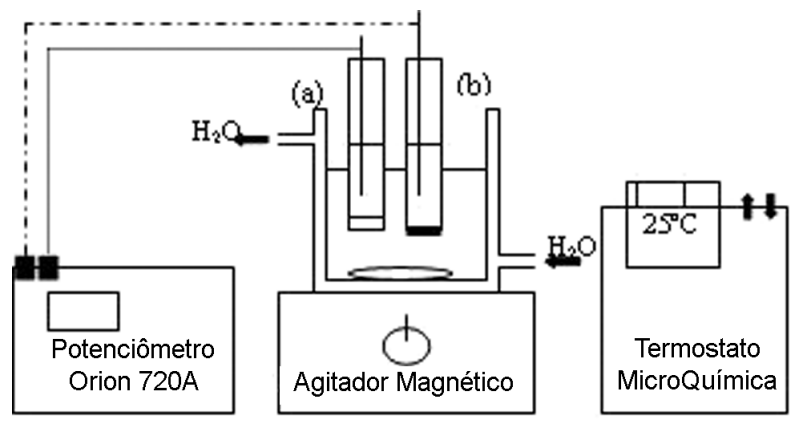

Figura 3. Esquema demonstrativo do sistema utilizado para realização das titulações: (a) eletrodo indicador de membrana seletiva para surfactante, (b) eletrodo de referência para brometo com solução interna

\section{RESULTADOS E DISCUSSÃO}

Mudanças nas propriedades da solução, quando do início da formação das micelas, orientam discussões envolvendo fenômenos de superfície e colóides. Assim, a cmc pode ser determinada pelo estudo de várias propriedades, como condutividade elétrica, tensão superficial e potencial, empregadas nesse estudo.

Em baixas concentrações, os monômeros de surfactantes encontram-se em equilíbrio em solução e adsorvidos nas interfaces. ${ }^{1}$ Com o aumento da concentração, a adsorção aumenta formando monocamada de surfactante, saturando a superfície da solução. Neste ponto, a adição de surfactantes provoca agregação da parte hidrofóbica das moléculas de surfactantes resultando em micelas, agregados de 50 ou mais monômeros, que adotam a forma esférica, conforme a Figura 1.

Acima da cmc não existe um aumento significativo no número de monômeros isolados e, conseqüientemente, a força de coesão das moléculas da superfície é pouco modificada, não se observando efeitos significativos sobre a tensão superficial da solução. No caso da condutividade elétrica, a partir da cmc o acréscimo é menor, pois os aglomerados micelares conduzem menos que os respectivos monômeros individuais.

A Figura 4 ilustra o perfil da curva condutimétrica. $\mathrm{O}$ ponto de encontro da extrapolação das duas retas corresponde à concentração micelar crítica $(\mathrm{cmc})$.

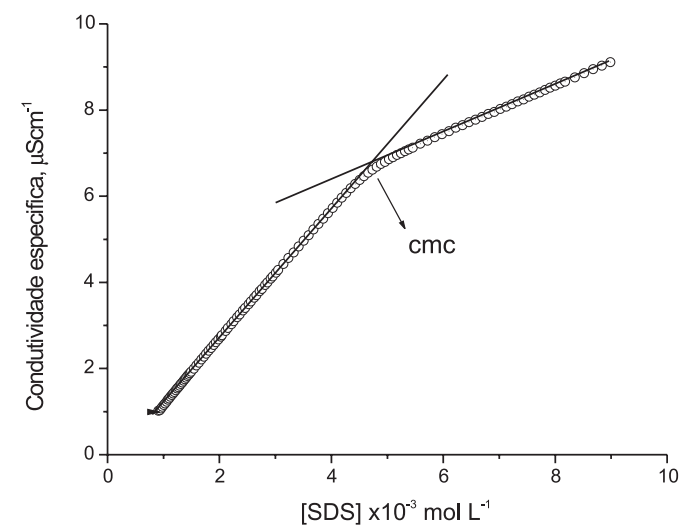

Figura 4. Curva da condutividade em função do log da concentração molar de SDS

Os surfactantes iônicos possuem dois tipos de comportamento em solução aquosa. Abaixo da cmc os monômeros comportam-se como eletrólito forte. Após a micelização, monômeros adicionados contribuem para a formação de micelas, diminuindo o incremento da condutividade da solução com a adição de surfactante, ${ }^{2}$ conforme ilustrado na figura acima.

Na Figura 5 tem-se o gráfico obtido para a medição da $\mathrm{cmc}$ utilizando tensiometria.

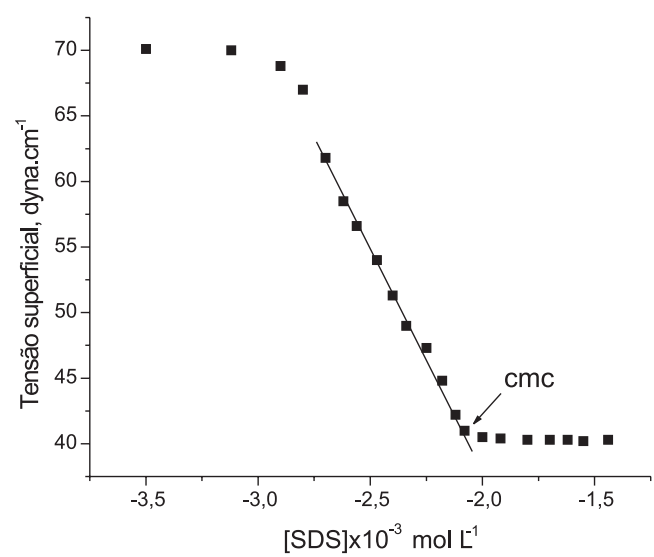

Figura 5. Curva da tensão superficial em função do log da concentração molar de SDS

Os líquidos tendem a adotar uma forma que minimize sua área de superfície, numa tentativa de manter as moléculas com um maior número possível de vizinhos semelhantes. ${ }^{15}$ As gotas de líquidos tendem a assumir a forma esférica, pois a esfera é a forma com a menor razão superfície/volume. Para se perturbar a superfície de um líquido é necessário realizar algum trabalho. A razão entre o trabalho realizado e a área de deslocamento na superfície do líquido é denominada tensão superficial. ${ }^{16}$ Este fenômeno confere ao líquido uma "casca" de moléculas na superfície, produto de um desequilíbrio das forças intermoleculares no líquido.

A tensão superficial da água é diminuída pela adição de surfactante. Quando um surfactante é adicionado à água, suas moléculas tendem a se arranjar de modo a minimizar a repulsão entre os grupos hidrofóbicos e a água. Próximo a superfície, os grupos polares orientam-se para a solução aquosa, enquanto que os grupos apolares se localizam na interface água-ar minimizando, assim, o contato com 
a água. Isto gera uma diminuição na tensão superficial do sistema, pois provoca um desarranjo de suas superfícies. Após a $\mathrm{cmc}$, ao contrário dos monômeros, as micelas se dispersam na solução, não apresentando efeito adicional sobre a tensão superficial.

Na Figura 6 é apresentado o perfil da curva de potencial em função da concentração de SDS.

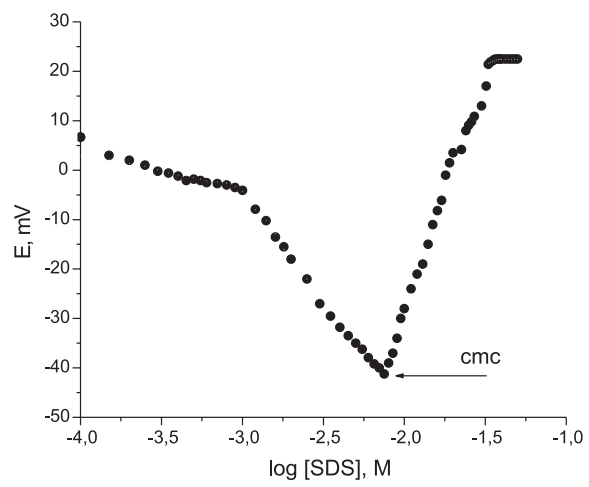

Figura 6. Curva do potencial em função do log da concentração molar de $S D S$

O perfil da curva mostra que a partir de aproximadamente 0,003 mol L ${ }^{-1} \mathrm{o}$ eletrodo se torna mais sensível à leitura do incremento de monômeros de SDS, mantendo um coeficiente angular bem definido. Com o aumento da concentração de SDS ocorre um decréscimo na diferença de potencial, observando-se um valor potenciométrico mínimo próximo a $0,0080 \mathrm{~mol} \mathrm{~L}^{-1}$. Esta inflexão caracteriza o início da formação de micelas, indicando a cmc do respectivo surfactante. Logo após a cmc, o acréscimo de surfactante produz um aumento na diferença de potencial. Tendo em vista que após a cmc, a concentração de monômeros do SDS permanece constante em solução, a variação da diferença de potencial pode ser atribuída ao aumento da concentração de agregados micelares na solução, que podem bloquear e diminuir a atividade dos monômeros na superfície do eletrodo.

$\mathrm{O}$ valor de cmc encontrado por potenciometria para SDS, neste trabalho, está em concordância com os valores experimentais apresentados na Tabela 1 e com a literatura. ${ }^{1,2}$

Tabela 1. Valores de cmc para dodecilsulfato de sódio (SDS), em mol L $\mathrm{L}^{-1}$, utilizando as técnicas condutimétrica, tensiométrica e potenciométrica

\begin{tabular}{cccc}
\hline & Condutimétrica & Tensiométrica & Potenciométrica \\
\hline $\mathrm{cmc}$ & $0,0084 \mathrm{~mol} \mathrm{~L}^{-1}$ & $0,0085 \mathrm{~mol} \mathrm{~L}^{-1}$ & $0,0080 \mathrm{~mol} \mathrm{~L}^{-1}$ \\
\hline
\end{tabular}

Determinou-se potenciometricamente a cmc do surfactante catiônico brometo de dodeciltrimetilamônio (DTAB), utilizando-se o eletrodo para surfactante construído em laboratório (Figura 7).

O perfil da curva ilustrada na Figura 7 apresenta quebra na linearidade em concentração próxima a $0,015 \mathrm{~mol} \mathrm{~L}^{-1}$, correspondente à cmc do surfactante. Após a cmc, a diferença de potencial tem um leve decréscimo e tende a um patamar. Devido à composição do eletrodo, que não possui internamente solução de DTAB, a sensibilidade do eletrodo diminui, não impedindo sua utilização na determinação da cmc deste surfactante. $\mathrm{O}$ valor encontrado está de acordo com o da literatura. ${ }^{17}$

A alteração brusca do comportamento do potencial, verificada nas Figuras 6 e 7, demonstra uma mudança das características da solução relacionada com a formação de micelas. Alteração essa que coincide com as das propriedades condutimétricas e tensiométricas evidenciando, assim, a coerência dos resultados obtidos nesse trabalho.

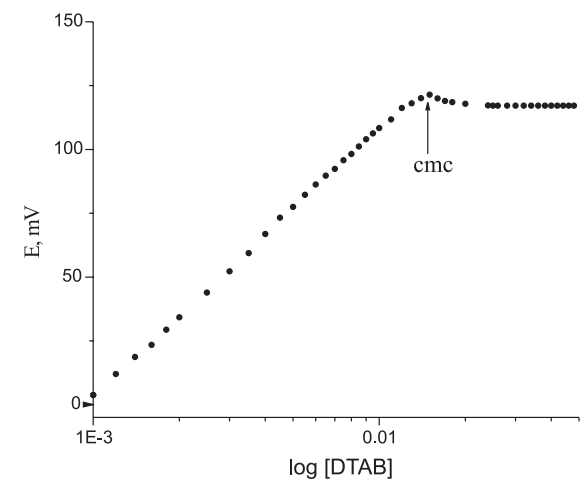

Figura 7. Curva do potencial em função do log da concentração molar de $D T A B$

\section{CONCLUSÕES}

O eletrodo de membrana para surfactante mostrou-se eficiente na determinação da cmc dos surfactantes aniônico e catiônico, sendo os valores encontrados para SDS e DTAB concordantes com os da literatura. ${ }^{2,10,13}$ Constatou-se, portanto, que a técnica potenciométrica é uma ferramenta adicional de baixo custo para o estudo de surfactantes em aulas práticas de graduação, no curso de Química, ampliando as possibilidades de aproveitamento de equipamentos existentes em laboratórios para o estudo de sistemas através de aplicação de diferentes técnicas de análise.

Os resultados aqui apresentados demonstram que técnicas fundamentadas em conceitos simples podem fornecer resultados bastante precisos na elucidação de sistemas surfactantes.

\section{AGRADECIMENTOS}

Depto de Química-UFSC/CNPq.

\section{REFERÊNCIAS}

1. Rizzatti, I. M.; Dissertação de Mestrado, Universidade Federal de Santa Catarina, Brasil, 2003.

2. Minatti, E.; Zanette, D.; Colloids Surf., B 1996, 113, 237.

3. Maniasso, N.; Quim. Nova 2001, 24, 87.

4. Rizzatti, I. M.; Tese de Doutorado, Universidade Federal de Santa Catarina, Brasil, 2007.

5. Ruzicka, J.; J. Chem. Educ. 1997, 74, 167.

6. Cattrall, R. W.; Freiser, H.; Anal. Chem. 1971, 43, 1905.

7. Frant, M. S.; Roos, J. W.; J. Sci. 1996, 154, 1533; Fricke, G. H.; Huntz, M. J.; J. Chem. Educ. 1977, 54, 517.

8. Martel, D.; Sojic, N.; Kuhn, A.; J. Chem. Educ. 2002, 79, 349.

9. Rizzzatti, I. M.; Zanette. D.; Zanette, D. R.; J. Braz. Chem. Soc. 2004, $15,491$.

10. Varela, N.; Martínez, A.; Enseñanza de las Ciencias 1997, 4, 151.

11. Demo, P.; Educar pela pesquisa, Autores Associados: Campinas, 1996.

12. Leach, J.; Practical work in school science: Which way now?, Routledge: London, 1998, cap. 4.

13. Silva, da S. F.; Núñez, I. B.; Quim. Nova 2002, 25, 1197.

14. Galiazzi, M. C.; Gonçalves, F. P.; Quim. Nova 2004, 27, 326.

15. Behring, J. L.; Lucas, M.; Machado, C.; Barcellos, I. O.; Quim. Nova 2004, 27, 492.

16. Russel, J. B.; Química Geral, 2a ed., Makron Books: São Paulo, 1994, vol. 2.

17. Dutschk, V.; Sabbatovskiy, K. G.; Stolz, M.; Grundke, K.; Rudoy, V. M.; J. Colloid Interface Sci. 2003, 267, 456. 